\title{
Alignments of galaxies and halos in hydrodynamical simulations
}

\author{
Isha Pahwa, ${ }^{1,2,3} \dagger$ and Noam I. Libeskind ${ }^{3}$ \\ ${ }^{1}$ Inter University Centre for Astronomy and Astrophysics, Post Bag 4, \\ Pune University Campus, Ganeshkhind, Pune 411007, India \\ ${ }^{2}$ Department of Physics and Astrophysics, University of Delhi, Delhi-110076 \\ ${ }^{3}$ Leibniz-Institut für Astrophysik Potsdam (AIP), An der Sternwarte 16, \\ D-14482 Potsdam, Germany
}

\begin{abstract}
We use a $200 h^{-1} \mathrm{Mpc}$ cosmological hydrodynamical simulation to examine the alignments of galaxies with respect to the host halo. We do separate study for the different components of the halo, such as stars, gas and dark matter. We show that angular momentum of gas is more aligned with the angular momentum of host halo compared with the stellar component.
\end{abstract}

Keywords. Galaxies: halos, Cosmology: theory, dark matter, large-scale structure of universe

\section{Introduction}

The origin of angular momentum is well known problem in the standard picture of cosmic structure formation. Current ideas of how dark matter halos get their spin, rely on torques imparted due to the misalignment of a collapsing Lagrangian region's inertia tensor and the tidal field (Peebles 1969; White 1984). Such torques are only effective in the linear regime, so long as the collapsing region has a large enough "lever arm". Halos (and the galaxies within them) acquire significant angular momentum in the non-linear stage of their evolution. Numerous numerical studies have dealt with the issues related to the non-linear angular momentum acquisition of dark matter halos, its evolution and its relation to the large-scale structure (LSS, Bett et al. 2010; Libeskind et al. 2012 among others). However, in the non-linear regime, baryons become important. Unlike dark matter, baryons are affected not only by gravity but also by hydrodynamic processes. These processes can dominate galaxy spin and hence, become important to study.

\section{Simulation}

We carry out a large cosmological hydrodynamical simulation CURIE, using the PMTreeSPH code GADGET-2 (Springel 2005), assuming the standard $\Lambda$ CDM cosmology (WMAP5). The cosmological parameters are these: $\Omega_{m}=0.28, \Omega_{\Lambda}=0.72, h=0.7=\mathrm{H}_{0} / 100$ $\mathrm{kms}^{-1} \mathrm{Mpc}^{-1}, \sigma_{8}=0.817$ and $n_{s}=0.96$. The simulation box size is $200 h^{-1} \mathrm{Mpc}$ with $2 \times 1024^{3}$ particles. The masses of dark matter, gas and star particles are $4.6 \times 10^{8} h^{-1} \mathrm{M}_{\odot}$, $9.7 \times 10^{7} h^{-1} \mathrm{M}_{\odot}$ and $4.85 \times 10^{7} h^{-1} \mathrm{M}_{\odot}$ respectively. The simulation uses the feedback and star formation rules of Springel \& Hernquist (2003). A halo catalogue is obtained by running the publicly available halo finder AHF (Knollmann \& knebe 2009) on the particle distribution. We use this simulation at $z=0$. 


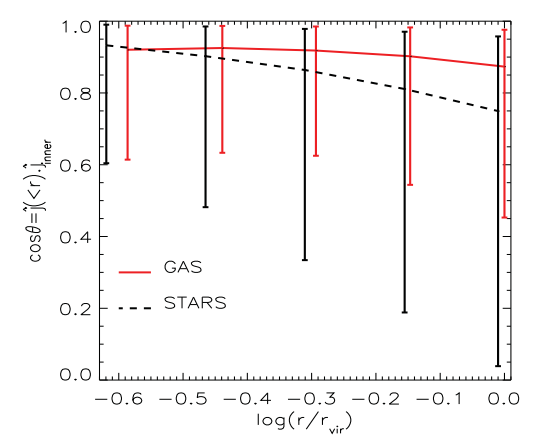

Figure 1. Orientation profiles of angular momentum of gas (or stars) in the inner region of halo with respect to the total angular momentum vector, $\hat{j}(<r)$ of the total halo as a function of radial distance.

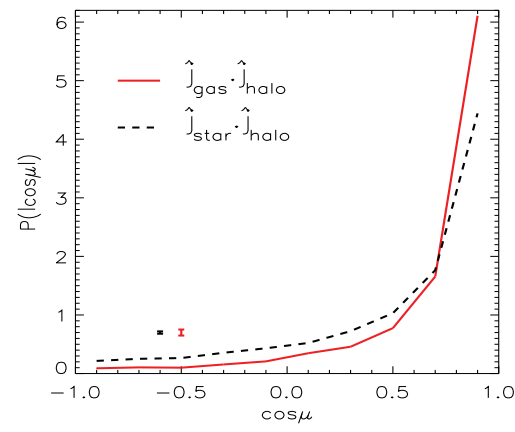

Figure 2. Probability distribution of the cosine of angle formed between the angular momentum vector of gas (or stars) in inner region of halos with total angular momentum of halo computed at virial radius.

\section{Results}

The study of orientations of the angular momentum of gas and star particles with respect to the total angular momentum of the halo is an important input in the formulation of models for galaxy formation. We define a spherical 'inner' region at $0.25 r_{\text {vir }}$ where $r_{\text {vir }}$ is the virial radius of the halo. Generally speaking, baryonic effects are dominant over dark matter in this region.

Around 25,000 halos in the mass range $10^{11.5}-10^{13} h^{-1} \mathrm{M}_{\odot}$ are selected for analysis. We ensure that each halo has at least 1000 baryonic particles within $0.25 r_{\text {vir }}$, such that the computed angular momentum directions are not affected by poor resolution (see Sales et al. 2012). Fig. 1 shows the (cosine of the) angle formed between the angular momentum of the gas or stellar component (red solid or black dashed line, respectively) computed at $0.25 r_{\text {vir }}$ with the angular momentum of the all material (i.e including dark matter) as a function of radius. At each radius, the median and $1 \sigma$ deviation are plotted. The angular momentum of the gas component in the inner parts of the halo shows a fairly strong alignment with the angular momentum of outer parts of the halo. The alignment of the stellar component with the outer parts of the halo, is not as strong, and the spread in alignment is larger, indicated by the increasing variance around medians.

In Fig. 2 we show the probability distribution of the $\cos \mu$, the cosine of the angle formed between the angular momentum of gas, $\hat{\mathrm{j}}_{\text {gas }}$ (red, solid line) or stars, $\hat{\mathrm{j}}_{\text {star }}$ (black, dash line) within the inner part of the halo with the total angular momentum of halo, $\hat{\mathrm{j}}_{\text {halo }}$ computed at $\mathrm{r}_{\mathrm{vir}}$. The small error bars in the lower left of the plot indicate the (Poissonian) scatter that one would expect from a uniform distribution of that size. As it is clear from this figure, $\hat{\mathrm{j}}_{\text {gas }}$ is more aligned with $\hat{\mathrm{j}}_{\text {halo }}$ than $\hat{\mathrm{j}}_{\text {star }}$ with $\hat{\mathrm{j}}_{\text {halo }}$.

IP is supported by the Deutsche Forschungs Gemeinschaft and the hospitality of LeibnizInstitut für Astrophysik Potsdam.

\section{References}

Bett, P., Eke, V., Frenk, C. S., Jenkins, A., \& Okamoto, T. 2010, MNRAS 404, 1137

Knollmann, S. R. \& Knebe, A. 2009, ApJS 182, 608

Libeskind, N. I., Hoffman, Y., Knebe, A., et. al. 2012, MNRAS 421, L137

Peebles, P. J. E. 1969, ApJ 155, 393

Sales, L. V., Navarro, J. F., Theuns, T., et. al. 2012, MNRAS 423, 1544

Springel, V. 2005, MNRAS 364, 1105

Springel, V. \& Hernquist, L. 2003, MNRAS 339, 289

White, S. D. M. 1984, ApJ 286, 38 